\title{
ERROS NA ADMINISTRAÇÃO DE MEDICAMENTOS: EVIDÊNCIAS E IMPLICAÇÕES NA SEGURANÇA DO PACIENTE
}

\author{
Andréa Tayse de Lima Gomes ${ }^{1}$, Yole Matias Silveira de Assis ${ }^{1}$, Micheline da Fonseca Silva ${ }^{1}$, Isabelle \\ Katherinne Fernandes Costa ${ }^{2}$, Alexsandra Rodrigues Feijão², Viviane Euzébia Pereira Santos ${ }^{2}$
}

\begin{abstract}
RESUMO: O estudo objetiva identificar as evidências e as implicações dos erros na administração de medicamentos na segurança do paciente. Trate-se de uma revisão integrativa, com busca dos estudos em quatro bases de dados, em maio de 2015. Obteve-se uma amostra de 40 artigos e foram submetidos à estatística descritiva. Houve predomínio de estudos descritivos - nível de evidência 4 ( $n=28 ; 70 \%$ ), publicados no Brasil $(n=17 ; 42,5 \%)$. Quanto aos erros, destacaram-se: erro de dosagem ( $n=27 ; 67,5 \%)$, medicação errada $(n=25 ; 62,5 \%)$; troca de paciente $(n=21 ; 52,5 \%)$; erro de horário $(n=20 ; 50 \%)$; via errada $(n=17 ; 42,5 \%)$, além de erro documental, omissão de justificativas quando necessário e outras. Este tipo de evento é responsável por deixar sequelas irreparáveis nos pacientes ou até levar à morte.
\end{abstract}

DESCRITORES: Erros de medicação; Segurança do paciente; Enfermagem.

\section{MEDICATION ADMINISTRATION ERRORS: EVIDENCES AND IMPLICATIONS FOR PATIENT SAFETY}

ABSTRACT: The objective is to identify the evidences and implications of medication administration errors for patient safety. An integrative review was undertaken through a search in four databases in May 2015. A sample of 40 articles was obtained, submitted to descriptive statistics. Descriptive studies of evidence level 4 were predominant $(n=28 ; 70 \%)$, published in Brazil ( $n=17 ; 42.5 \%)$. The following errors stood out: dose errors $(n=27 ; 67.5 \%)$, wrong drug $(n=25 ; 62.5 \%)$; wrong patient $(n=21 ; 52.5 \%)$; wrong time $(n=20 ; 50 \%)$; wrong route $(n=17 ; 42.5 \%)$, besides documentation errors, omission of justifications when necessary and others. This type of event is responsible for causing irreparable damage to the patient or even death.

DESCRIPTORS: Medication errors; Patient safety; Nursing.

\section{ERRORES EN LA ADMINISTRACIÓN DE MEDICAMENTOS: EVIDENCIAS Y IMPLICACIONES EN LA SEGURIDAD DEL PACIENTE}

RESUMEN: La finalidad del estudio es identificar las evidencias y las implicaciones de los errores en la administración de medicamentos en la seguridad del paciente. Se trata de una revisión integradora con búsqueda de estudios en cuatro bases de datos en mayo del 2015. Fue encontrada una muestra de 40 artículos, sometidos a la estadística descriptiva. Predominaron estudios descriptivos - nivel de evidencia 4 ( $n=28 ; 70 \%)$, publicados en Brasil $(n=17 ; 42,5 \%)$. Entre los errores se destacaron: error de dosificación ( $n=27 ; 67,5 \%)$, medicación equivocada $(n=25 ; 62,5 \%)$; cambio de paciente $(n=21 ; 52,5 \%)$; error de horario $(n=20 ; 50 \%)$; vía equivocada ( $n=17 ; 42,5 \%)$, además de error documental, omisión de justificativas cuando necesario y otras. Este tipo de evento es responsable por dejar secuelas irreparables en los pacientes o incluso llevar a la muerte.

DESCRIPTORES: Errores de medicación; Seguridad del paciente; Enfermería.

${ }^{1}$ Enfermeira. Mestranda do Programa de Pós-Graduação em Enfermagem da Universidade Federal do Rio Grande do Norte. Natal, RN, Brasil.

${ }^{2}$ Enfermeira. Doutora em Enfermagem. Docente do Departamento de Enfermagem e Pós Graduação em Enfermagem da Universidade Federal do Rio Grande do Norte. Natal, RN, Brasil. 


\section{INTRODUÇÃO}

No ano de 2004, a Organização Mundial da Saúde instituiu a Aliança Mundial para a Segurança do Paciente, um programa cujo princípio básico consiste em estabelecer assistência à saúde segura, a partir do desenvolvimento de políticas públicas por parte dos Estados membros ${ }^{(1)}$.

O termo Segurança do Paciente (SP) foi definido pelo Ministério da Saúde através da Portaria $\mathrm{n}^{\circ}$ 529, de $1^{\circ}$ de abril de 2013, como a "redução a um mínimo aceitável do risco de dano desnecessário associado ao cuidado em saúde". Este dano diz respeito ao comprometimento de estruturas ou funções do organismo humano, seja físico, social ou psicológico ${ }^{(2)}$.

Esta portaria institui o Programa Nacional de Segurança do Paciente (PNSP), que tem por objetivo contribuir para a prestação de cuidado de qualidade nos serviços de saúde do Brasil. Neste contexto, foram desenvolvidos seis protocolos básicos voltados para a SP, implantados junto ao PNSP, a saber: identificação do paciente; comunicação efetiva entre os profissionais de saúde; segurança na prescrição, uso e administração de medicamentos; cirurgia segura; higienização das mãos; minimizar risco de quedas e úlceras por pressão ${ }^{(2)}$.

Dentre os protocolos supracitados, o presente estudo enfatiza a Administração de Medicamentos (AM), que consiste numa ação complexa que envolve diversos profissionais de saúde e deve ser realizada de forma segura, com vistas a valorizar a qualidade desta técnica, de modo a reduzir a ocorrência de possíveis Eventos Adversos (EA) ${ }^{(3)}$.

Deste modo, os EA dizem respeito aos incidentes evitáveis que produzem agravos ao paciente. Estes erros acontecem frequentemente nos serviços de saúde e, em geral, implicam em consequências graves para o paciente e/ou para o profissional. Devido a esta realidade, os EA conformam-se em um problema de saúde pública ${ }^{(2,4)}$.

Logo, o cuidado seguro em relação à AM requer que os profissionais detenham conhecimentos sólidos e embasados na literatura científica específica quanto aos aspectos intrínsecos às medicações e a sua administração, como: reações medicamentosas e técnicas de administração, respectivamente. Além disto, este procedimento demanda responsabilidade e atenção na realização de todas as etapas deste processo pelos profissionais de saúde, em especial pela equipe de enfermagem, visto ser esta a principal responsável por tal prática ${ }^{(4)}$.

Diante do exposto, denota-se que a administração de medicamentos constitui-se em uma prática de fundamental importância para a SP. Destarte, é imprescindível a abordagem desta temática no âmbito da pesquisa, vislumbrando a publicação de estudos que subsidiem a prática baseada em evidências em busca do cuidado seguro e de qualidade. Portanto, elaborou-se a seguinte questão de pesquisa: quais os aspectos abordados na literatura científica sobre erros na administração de medicamentos?

Nesse sentido, o presente estudo objetivou identificar as evidências e implicações dos erros na administração de medicamentos na segurança do paciente.

\section{- MÉTODOS}

Trata-se de uma revisão integrativa de literatura, que consiste na síntese de pesquisas prévias e, sobretudo, descreve as conclusões do corpus da literatura sobre um fenômeno específico, cuja execução desenvolve-se em cinco etapas, a saber: 1) formulação do problema de pesquisa, estabelecimento dos objetivos da revisão e critérios de seleção para o arrolamento dos artigos; 2) definição das variáveis a serem extraídas das publicações; 3 ) seleção e avaliação dos artigos nas bases de dados; 4) análise dos resultados; e, 5) discussão e apresentação dos dados ${ }^{(5-6)}$.

A fim de nortear a busca dos artigos nas bases de dados, elaborou-se um protocolo composto pelos seguintes tópicos: temática; objetivo; questão norteadora; estratégia para a coleta de dados; critérios para elegibilidade dos estudos; dados a serem coletados para avaliação crítica dos estudos; síntese e análise dos dados. 
A busca pelos estudos foi realizada nas bases de dados Literatura Latino-Americana e do Caribe em Ciências da Saúde (LILACS), National Library of Medicine (PubMed Central), Scopus Info Site (SCOPUS) e Base de Dados de Enfermagem (BDENF), no mês de maio de 2015.

Para o refinamento apropriado dos estudos selecionados, definiu-se uma amostra conforme os critérios de inclusão preestabelecidos, a saber: artigos disponíveis gratuitamente e em texto completo nas bases de dados supracitadas, sem limite definido de tempo da publicação desde que os artigos respondessem a questão de pesquisa. Foram excluídas as publicações que versavam sobre a $A M$ em crianças e recém-nascidos e estudos em formato de editoriais, carta ao editor, dissertações, teses, artigos de opinião e outras revisões integrativas da literatura.

O arrolamento dos artigos na LILACS e na BDENF deu-se mediante a utilização dos descritores indexados dos Descritores em Ciências da Saúde (DeCS): Erros de Medicação, Segurança do Paciente e Enfermagem. Nas bases de dados PUBMED e SCOPUS foram empregados os descritores indexados do vocabulário MeSH - Medical Subject Headings, na língua inglesa: Medication Errors, Patient Safety e Nursing.

O cruzamento entre os referidos descritores ocorreu através do operador booleano AND e a busca nas bases de dados anteriormente mencionadas aconteceu de forma não controlada, assim como foi usado o recurso de busca ", a fim de limitar a pesquisa apenas aos termos que estivessem adjacentes nos artigos encontrados. Sendo assim, as estratégias de buscas utilizadas nas bases de dados estão expostas na Figura 1.

Após o processo de busca dos estudos nas bases de dados, as publicações foram pré-selecionadas com base na leitura dos títulos e resumos. Em seguida, procedeu-se a leitura na íntegra dos artigos previamente selecionados e, finalmente, foram eleitas as publicações que compuseram a amostra final dessa pesquisa.

Na Tabela 1 encontra-se a representação do processo de seleção dos estudos que constituíram o número de artigos encontrados em cada base de dados e a amostra de estudos pré-selecionados para a leitura na íntegra, de acordo com as estratégias de busca utilizadas nos bancos de dados eletrônicos.

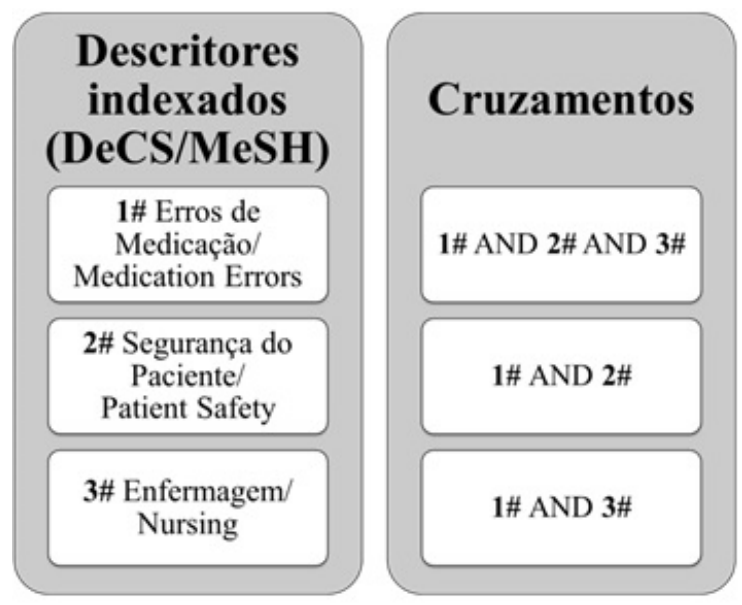

Figura 1 - Descritores e estratégias para a busca dos artigos nas bases de dados. Natal, RN, Brasil, 2015

Tabela 1 - Distribuição do processo de seleção dos artigos. Natal, RN, Brasil, 2015

\begin{tabular}{lccccc} 
& \multicolumn{3}{c}{ Resultado da pesquisa } & $\begin{array}{c}\text { Estudos pré- } \\
\text { selecionados* }\end{array}$ \\
\cline { 2 - 5 } & 1\# AND 2\# AND 3\# & 1\# AND 2\# & 1\# AND 3\# & $\mathbf{n}$ \\
\cline { 2 - 5 } & $\mathbf{n}$ & $\mathbf{n}$ & 31 & 80 & 29 \\
\hline LILACS & 20 & 337 & 190 & 18 \\
\hline PUBMED & 56 & 463 & 101 & 29 \\
\hline SCOPUS & 216 & 14 & 66 & 10 \\
\hline BDENF & 11 & $\mathbf{8 4 5}$ & $\mathbf{4 3 7}$ & $\mathbf{8 6}$
\end{tabular}

*Estudos selecionados para a leitura na íntegra, conforme critérios de elegibilidade. 
A Figura 2 corresponde ao fluxograma referente ao processo de seleção dos artigos que integraram a amostra final do presente estudo, de acordo com as etapas de seleção das publicações.

Para a coleta de dados, utilizou-se um instrumento elaborado pelos próprios pesquisadores e aplicado em pesquisas anteriores da mesma natureza, composto pelas seguintes variáveis: bases de dados; título do artigo; país onde foi realizado o estudo; idioma; ano de publicação; local (hospital ou atenção básica); se hospital, setor; desenho metodológico (tipo de estudo); nível de evidência; descrição do erro na AM.

A classificação das evidências científicas dos artigos analisados deu-se consoante aos níveis de evidências recomendado pelo Joanna Briggs Institute ${ }^{(7)}$, os quais são: nível 1 - revisões sistemáticas e ensaios clínicos randomizados; nível 2 - revisões sistemáticas de estudos quase experimentais, estudos prospectivos controlados quase experimentais e estudos retrospectivos com grupo controlado; nível 3 - revisões sistemáticas envolvendo estudos de coorte, estudos de coorte e estudos de caso-controle; nível 4 - revisões sistemáticas de estudos descritivos, estudos seccionais, séries de casos e estudo de caso; nível 5 - revisões sistemáticas de opiniões de especialistas, consensos de especialistas e bancos de investigações ou opinião de um único especialista.

Por fim, após a leitura flutuante e análise crítica dos artigos selecionados, os resultados foram digitados em planilhas eletrônicas no programa Microsoft Excel 2010 ${ }^{\circledR}$, analisados por estatística descritiva e apresentados em forma de Quadro e Tabela.

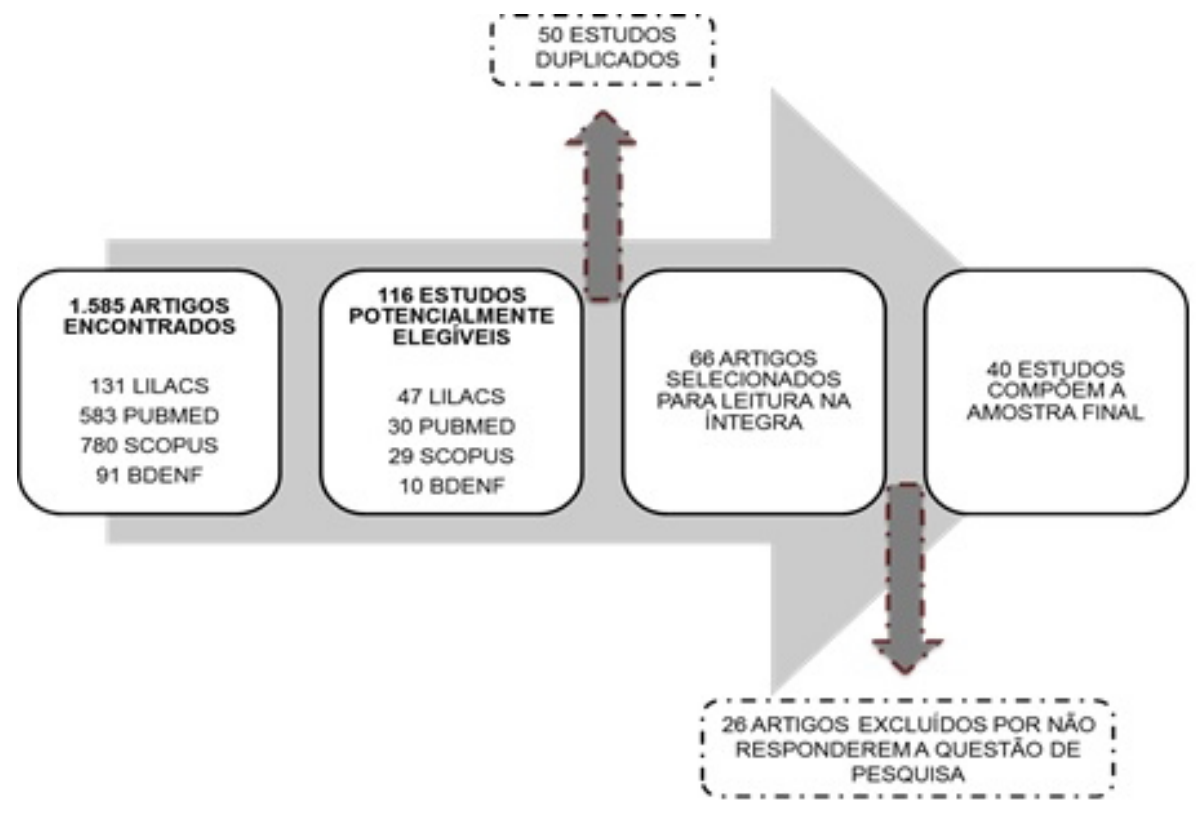

Figura 2 - Fluxograma relacionado ao processo de seleção dos artigos. Natal, RN, Brasil, 2015

\section{RESULTADOS}

A amostra desse estudo compreendeu 40 artigos, cuja caracterização encontra-se descrita no Quadro 1 de acordo com o país onde o estudo foi publicado, o tipo de estudo, com suas respectivas referências, e a classificação do nível de evidência.

Conforme explicitado no Quadro1, houve predomínio dos estudos executados no Brasil ( $\mathrm{n}=17 ; 42,5 \%)$, seguido por Estados Unidos ( $n=6 ; 15 \%)$. Entre os tipos de estudo, destacaram-se os descritivos - Nível de evidência $4(n=28 ; 70 \%)$ e os observacionais - Nível de evidência 3 ( $n=9 ; 22,5 \%)$.

Na Tabela 2 encontra-se a distribuição dos tipos de erros no processo de AM de acordo com as estatísticas descritivas correspondentes (n e \%).

De acordo com o exposto na Tabela 2, observou-se que o maior número de erros alusivos à AM está relacionado à dose $(n=27 ; 67,5 \%)$, seguido por erros de medicação $(n=25 ; 62,5 \%)$. No entanto, os 
erros atrelados ao volume administrado e à distração dos profissionais durante a administração dos fármacos foram reportados apenas em um (2,5\%) artigo.

Quadro 1 - Caracterização dos estudos incluídos na amostra final da pesquisa. Natal, RN, Brasil, 2015

\begin{tabular}{|l|l|c|}
\hline País & Tipo de estudo & Nível de evidência \\
\hline \multirow{2}{*}{ Brasil $(\mathrm{n}=17 ; 42,5 \%)$} & Observacional & 3 \\
\cline { 2 - 3 } & Descritivo & 4 \\
\hline \multirow{2}{*}{ Austrália $(\mathrm{n}=3 ; 7,5 \%)$} & Observacional & 3 \\
\cline { 2 - 3 } & Descritivo & 4 \\
\hline Bélgica $(\mathrm{n}=1 ; 2,5 \%)$ & Observacional & 3 \\
\hline Coreia do Sul $(\mathrm{n}=1 ; 2,5 \%)$ & Descritivo & 3 \\
\hline \multirow{3}{*}{ Estados Unidos $(\mathrm{n}=6 ; 15 \%)$} & Observacional & 4 \\
\cline { 2 - 3 } & Descritivo & 4 \\
\hline Finlândia $(\mathrm{n}=1 ; 2,5 \%)$ & Descritivo & 4 \\
\hline Irã $(\mathrm{n}=2 ; 5 \%)$ & Descritivo & 4 \\
\hline Irlanda $(\mathrm{n}=1 ; 2,5 \%)$ & Descritivo & 4 \\
\hline Israel $(\mathrm{n}=1 ; 2,5 \%)$ & Descritivo & 2 \\
\hline Singapura $(\mathrm{n}=1 ; 2,5 \%)$ & Descritivo & 4 \\
\hline Canadá $(\mathrm{n}=2 ; 5 \%)$ & Experimental & 2 \\
\cline { 2 - 3 } & Descritivo & 3 \\
\hline China $(\mathrm{n}=1 ; 2,5 \%)$ & Experimental & 3 \\
\hline França $(\mathrm{n}=1 ; 2,5 \%)$ & Observacional & 1 \\
\hline Holanda $(\mathrm{n}=1 ; 2,5 \%)$ & Observacional & 4 \\
\hline Inglaterra $(\mathrm{n}=1 ; 2,5 \%)$ & Revisão sistemática & \\
\hline
\end{tabular}

Tabela 2 - Distribuição dos tipos erros encontrados nas publicações analisadas. Natal, RN, Brasil, 2015

\begin{tabular}{lcc} 
Tipo de erro & $\mathbf{n}$ & ** $^{*}$ \\
\hline Dose errada & 27 & 67,5 \\
\hline Medicação errada & 25 & 62,5 \\
\hline Paciente errado & 21 & 52,5 \\
\hline Horário errado & 20 & 50 \\
\hline Via errada & 17 & 42,5 \\
\hline Erro documental & 16 & 40 \\
\hline Omissão na administração do medicamento & 14 & 35 \\
\hline Técnica incorreta & 13 & 32,5 \\
\hline Não observação dos profissionais quanto a possíveis reações medicamentosas & 6 & 15 \\
\hline Velocidade errada & 4 & 10 \\
\hline Medicamento vencido & 2 & 5 \\
\hline Volume errado & 1 & 2,5 \\
\hline Distração durante a administração & 1 & 2,5
\end{tabular}

*O percentual (\%) foi calculado com base no total de artigos analisados $(n=40)$

\section{DISCUSSÃO}

Denotou-se, a partir dos resultados, que a preocupação com os EA em saúde decorrentes da AM é uma inquietação mundial, apesar de haver destaque para o cenário brasileiro. Entretanto, a 
concentração de publicações de estudos realizados no Brasil pode estar relacionada às características das bases de dados eleitas na busca eletrônica.

Ademais, constatou-se que estudos descritivos $(\mathrm{n}=28 ; 70 \%)^{(8-35)}$ publicados no âmbito nacional $(\mathrm{n}=17 ; 42,5 \%)^{(8-22,36-37)}$ predominaram nessa pesquisa. Diante disto, embora haja uma propensão de se considerar estudos descritivos como estudos "menores" ou de uso restrito, pois deles não se poderia fazer nenhum tipo de inferência, estes podem ser uma ferramenta de gestão extremamente importante em sistemas de saúde, de forma a contribuir substancialmente para a prática baseada em evidências ${ }^{(38)}$.

Entretanto, os estudos descritivos estão classificados no nível de evidência $4^{(7)}$, configurando-se em baixa confiabilidade para generalização dos dados e aplicação prática. Portanto, recomenda-se o investimento em pesquisas experimentais (nível de evidência 2) ${ }^{(7)}$ a fim de proporcionar maior credibilidade para a utilização na esfera assistencial e gerencial do cuidar em saúde.

Não obstante, ressalta-se que a escolha de uma determinada abordagem na tentativa de prover uma resposta a um dado problema de pesquisa ou hipótese deve sempre considerar a realidade do pesquisador, os recursos disponíveis, bem como a aplicabilidade destes resultados no campo prático ${ }^{(38)}$.

Deste modo, é notório que a qualidade e a abordagem metodológica dos estudos influenciam diretamente na aplicabilidade da práxis dos profissionais de enfermagem nos serviços de saúde frente à SP no processo de administração de medicamentos.

Destarte, sabe-se que a AM é uma das principais atribuições da equipe de enfermagem e esta realidade exige amplo conhecimento acerca desta temática a fim de contribuir para a SP na realização do referido procedimento, de forma a prestar o cuidado livre de imperícia, negligência e imprudência. Assim, é incumbência do enfermeiro o planejamento das ações de enfermagem, de modo a serem minimizados os danos ao paciente advindos de erros relacionados à $\mathrm{AM}^{(39)}$.

Neste ínterim, autores ${ }^{(40-41)}$ descrevem possíveis causas para a ocorrência de erros relacionados à AM, a saber: sobrecarga de trabalho, associado à rotina intensa de trabalho e baixa remuneração; falta de atenção dos profissionais; inexperiência na prática assistencial, em geral, em referência à formação deficiente oferecida por algumas instituições de ensino; e estrutura inadequada oferecida pelos serviços de saúde.

Dentre os tipos de erros na $\mathrm{AM}$ encontrados nesta revisão, o principal deles diz respeito à dosagem medicamentosa, presente em 27 estudos $(67,5 \%)^{(8,10-11,13-14,16-17,19-20,23-28,30-32,34,36,42-49)}$. Corroborando com tal achado, uma pesquisa ${ }^{(50)}$ que teve como um dos objetivos identificar o perfil dos erros cometidos durante o preparo, encontrou que $67,7 \%$ dos erros estavam associados a doses preparadas erroneamente.

Neste contexto, para que a equipe de enfermagem administre a dosagem correta, é necessário que o profissional conheça o medicamento, as unidades que o compõem e certificar-se se a droga que foi prescrita está dentro do intervalo de dose conhecido. Além disso, é imprescindível que os profissionais de enfermagem tenham conhecimento sobre cálculo de medicação, o qual deve ser cautelosamente executado para não causar administração de doses equivocadas ${ }^{(51)}$.

Conforme dados publicados pelo Conselho Regional de Enfermagem de São Paulo em associação com a Rede Brasileira de Enfermagem e Segurança do Paciente, os dois tipos mais frequentes de erros relacionados à AM nos serviços de saúde são medicamento errado e troca de paciente(52). Esta realidade também foi encontrada na presente pesquisa, em que a administração da medicação errada foi o segundo erro mais comum entre os estudos analisados e esteve presente em $62,5 \%(n=25)^{(8-}$ $11,13,16,20-21,23-26,28-31,36-37,42-43,45-46,48-49,53)$ da amostra, seguido pelo medicamento administrado ao paciente errado $(\mathrm{n}=21 ; 52,5 \%)^{(8-11,16,18,22,23-28,30-31,33-34,36-37,46-47)}$.

A fim de minimizar este tipo de evento, enfatiza-se a importância de os profissionais de saúde verificarem a identificação dos pacientes antes da realização de qualquer procedimento. A utilização de placas de identificação dos pacientes, em locais visíveis à equipe, e de pulseiras de identificação, também são notáveis medidas na prevenção de erros na $\mathrm{AM}^{(20)}$.

No tocante ao horário prescrito, insta considerar que o fármaco deve ser administrado no horário certo com o intuito de garantir os níveis séricos terapêuticos ${ }^{(54)}$. No entanto, esse estudo mostrou que $50,0 \%(n=20)^{(9-10,12-17,20,23,42,25,27,30,34,36-37,47-49)}$ dos erros na AM estão associados ao erro de horário. 
Diante deste cenário, ressalta-se que o profissional deve respeitar o horário correto para a administração da droga, sem ultrapassar o limite de trinta minutos após o horário aprazado, pois, caso isso ocorra, a biodisponibilidade do medicamento será afetada ${ }^{(54)}$.

Entretanto, o principal fator que contribui ao erro e que afeta a administração das medicações no horário correto está relacionado à deficiência dos recursos humanos disponíveis nas próprias instituições de saúde, realidade esta que se atrela à sobrecarga de trabalho dos profissionais ${ }^{(41,51)}$.

Em relação à AM pela via errada, essa pesquisa demonstrou que 42,5\% $(n=17)^{(8-11,13,16,18,21-23,25,27,30-31,36-37,42)}$ dos artigos analisados abordavam este erro. Os erros de via são frequentes nos serviços hospitalares em todo o mundo e sabe-se que essas situações podem resultar em EA severos aos usuários, incluindo morte $^{(55)}$.

Diante disto, pesquisadores ${ }^{(55)}$ verificaram que os itens da prescrição médica podem contribuir consideravelmente para os erros de via. Posto que, na pesquisa supracitada, constatou-se que $91,3 \%$ das prescrições continham siglas/abreviaturas não padronizadas, 22,8\% não continham dados dos usuários e 4,3\% não apresentavam data e continham rasuras. Estas falhas documentais contribuem substancialmente para os erros atrelados à AM, incluindo erros de via, de paciente, de dose e de medicamento, de modo a gerar sequelas graves e até mesmo óbito dos pacientes.

Adiciona-se a isto a importância da anotação correta nos registros de enfermagem, que deve ser realizada após a AM. A execução desta anotação antes da medicação é um risco, posto que o paciente possa recusar a droga, assim como o esquecimento de registrar pode causar uma nova administração do fármaco por outro profissional, resultando em danos e agravos à saúde do paciente ${ }^{(51)}$. Apesar deste fato, o presente estudo verificou que $40,0 \%(n=16)^{(10,12,21-22,24-25,27-31,33,35-36,43,56)}$ dos artigos analisados abordaram a ocorrência do erro documental no processo de AM.

Por conseguinte, sugere-se que, durante o preparo da medicação, o profissional de enfermagem utilize como forma de prevenir o erro a identificação de cada medicamento com etiquetas, incluindo o leito, nome do paciente, droga, dose, via de administração e horário. E, após a AM, é de suma importância a checagem da medicação e justificativa, caso o profissional não tenha feito a administração ${ }^{(51)}$.

Desta maneira, se não houver a justificativa alusiva a não AM prescrito, explicitada nos registros documentais do paciente, o ato será caracterizado como omissão. E, nessa pesquisa, evidenciou-se que $14(35,0 \%)^{(13-15,17,19-20,23-24,36,44-45,47-49)}$ estudos relataram este tipo de erro.

Uma vez finalizada a administração do fármaco, o profissional deve monitorar o paciente quanto a ocorrências de possíveis reações adversas e constatar se o mesmo está tendo a resposta esperada. Neste aspecto, o profissional deve conhecer a ação da droga e saber distingui-la dos eventos adversos $^{(51)}$. Mesmo diante desta recomendação, a presente revisão observou que seis $(15,0 \%)^{(11,26-27,30-}$ ${ }^{31,33)}$ publicações discorreram sobre a não observância dos profissionais no que se refere às reações medicamentosas nos pacientes.

Sabe-se que os profissionais de enfermagem têm habilidade legal e técnica para realizar o preparo e AM, contudo devem ser observados os requisitos básicos que garantam a ausência de danos ao paciente, conforme o preconizado pelo Código de Ética dos profissionais de Enfermagem ${ }^{(57)}$.

É neste sentido que pesquisadores ${ }^{(37,51,54)}$ estão interessados na compreensão dos fatores que propiciem o erro e nas medidas preventivas, buscando estratégias a serem implementadas nos serviços de saúde, vislumbrando a minimização dos EA atrelados ao preparo e AM, como: dupla verificação da prescrição; certificação dos nove certos; notificação de erros; apoio de sistemas eletrônicos; sistema efetivo de registros; dose unitária; educação continuada dos profissionais; ambiente físico bem estruturado; e boa organização do trabalho.

Apesar de os artigos analisados responderem a questão de pesquisa com exímio, esse estudo apresentou a seguinte limitação: grande parte das publicações é descritiva e, portanto, enquadra-se em baixo nível de evidência, dificultando a aplicação prática dos resultados nos serviços de saúde.

\section{- CONCLUSÃO}


A análise dos estudos selecionados permitiu constatar que, apesar de haver inúmeras publicações acerca da temática estudada, ainda é notória a deficiência na qualidade metodológica para a aplicação do conhecimento produzido na prática, posto que a maioria dos estudos é do tipo descritivo e, consequentemente, enquadra-se em um baixo nível de evidência. Esta realidade reflete a necessidade de um maior investimento em pesquisas experimentais, com vistas a maior confiabilidade das publicações para aplicação na prática baseada em evidências.

Além disso, entre os artigos incluídos na amostra final dessa revisão, a dose e a medicação erradas, a troca de paciente e o erro de horário foram as falhas mais recorrentes atreladas ao processo de AM nos serviços de saúde. Assim, o grande número de estudos encontrados acerca do assunto denota a veemente preocupação dos pesquisadores, profissionais de saúde e gestores quanto a este tipo de evento. Afinal, estes incidentes são responsáveis por causar sequelas irreparáveis e até mesmo a morte.

Portanto, é imprescindível que os profissionais de saúde busquem a qualificação e atualização periodicamente, a fim de minimizar os eventos adversos advindos deste procedimento, vislumbrando a contribuição para o cuidado seguro e de qualidade.

\section{- REFERÊNCIAS}

1. World Health Organization (WHO). APPS web-based registration mechanism open. [Internet] Geneva: WHO; 2012 [acesso em 20 mai 2015]. Disponível: http://www.who.int/patientsafety/en/.

2. Ministério da Saúde (BR). Portaria n. 529 do Ministério da Saúde, de $1^{\circ}$ de abril de 2013. Institui o Programa Nacional de Segurança do Paciente (PNSP). Diário Oficial da União, Brasília, 02 abr. 2013.

3. Silva RP, Barreto BMF, Tenório DM, Camacho ACLF, de Oliveira BGRB. Revisão integrativa sobre administração de medicamentos na assistência de enfermagem. R. pes.: cuid. fundam. online. [Internet] 2013; 5(5) [acesso em 01 abr 2015]. Disponível: http://www.seer.unirio.br/index.php/cuidadofundamental/article/view/1581/pdf_978.

4. Vestena CFL, Girardon-Perlini NMO, da Rosa BVC, Stamm B, Beuter M, da Rosa N. Erros na administração de medicamentos: estudo com uma equipe de enfermagem. Rev. Enferm. UFPI. [Internet] 2014;3(4) [acesso em 01 abr 2015]. Disponível: http://revistas.ufpi.br/index.php/reufpi/article/view/2293/pdf.

5. Whittemore R, Knafl K. The integrative review: updated methodology. J. Adv. Nurs. 2005; 52(5): 546-53.

6. de Souza MT, da Silva MD, de Carvalho R. Revisão integrativa: o que é e como fazer. Einstein. [Internet] 2010; 8(1) [acesso em 01 abr 2015]. Disponível: http://astresmetodologias.com/material/O_que_e_RIL.pdf.

7. Joanna Briggs Institute (JBI). Levels of Evidence 2013. [Internet] 2013 [acesso em 01 abr 2015]. Disponível: http://joannabriggs.org/jbi-approach.html\#tabbed-nav=Levels-of-Evidence.

8. de Carvalho VT, Cassiani SHB, Chiericato C, Miasso AI. Erros mais comuns e fatores de risco na administração de medicamentos em unidades básicas de saúde. Rev. Latino-Am. Enfermagem. [Internet] 1999; 7(5) [acesso em 04 mai 2015]. Disponível: http://dx.doi.org/10.1590/S0104-11691999000500009.

9. Corbellini VL, Schilling MCL, Frantz SF, Godinho TG, Urbanetto JS. Eventos adversos relacionados a medicamentos: percepção de técnicos e auxiliares de enfermagem. Rev. bras. enferm. [Internet] 2011; 64(2) [acesso em 04 mai 2015]. Disponível: http://dx.doi.org/10.1590/S0034-71672011000200004.

10. Dias JD, Mekaro KS, Tibes CMS, Zem-Mascarenhas SH. Compreensão de enfermeiros sobre segurança do paciente e erros de medicação. Reme, Rev. Min. Enferm. [Internet] 2014; 18(4) [acesso em 04 mai 2015]. Disponível: http://www.dx.doi.org/10.5935/1415-2762.20140064.

11. Praxedes MFS, Telles Filho PCP. Erros e ações praticadas pela instituição hospitalar no preparo e administração de medicamentos. Reme, Rev. Min. Enferm. [Internet] 2011; 15(3) [acesso em 04 mai 2015]. Disponível:

http://www.reme.org.br/artigo/detalhes/52.

12. Rodrigues MCS, Oliveira LC. Erros na administração de antibióticos em unidade de terapia intensiva de hospital de ensino. Rev. Eletr. Enf. [Internet] 2010; 12(3) [acesso em 04 mai 2015]. Disponível:

http://dx.doi.org/10.5216/ree.v12i3.11935. 
13. Toffoletto MC, Padilha KG. Consequências de medicação em unidades de terapia intensiva e semi-intensiva. Rev. esc. enferm. USP. [Internet] 2006; 40(2) [acesso em 04 mai 2015]. Disponível: http://dx.doi.org/10.1590/S0080-62342006000200013.

14. Bohomol E, Ramos LH. Erro de medicação: importância da notificação no gerenciamento da segurança do paciente. Rev. bras. enferm. [Internet] 2007; 60(1) [acesso em 04 mai 2015]. Disponível: http://dx.doi.org/10.1590/S0034-71672007000100006.

15. Silva AEBC, Cassiani SHB. Análise prospectiva de risco do processo de administração de medicamentos antiinfecciosos. Rev. Latino-Am. Enfermagem. [Internet] 2013; 21(n.esp) [acesso em 04 mai 2015]. Disponível: http://dx.doi.org/10.1590/S0104-11692013000700029.

16. Marques TC, Reis AMM, Silva AEBC, Gimenes FRE, Opitz SP, Teixeira TCA, et al. Erros de administração de antimicrobianos identificados em estudo multicêntrico brasileiro. Rev. Bras. Ciênc. Farm. [Internet] 2008; 44(2) [acesso em 04 mai 2015]. Disponível: http://dx.doi.org/10.1590/S1516-93322008000200016.

17. Franco JN, Ribeiro G, D'innocenzo M, Barros BPA. Percepção da equipe de enfermagem sobre fatores causais de erros na administração de medicamentos. Rev. bras. enferm. [Internet] 2010; 63(6) [acesso em 04 mai 2015 ]. Disponível: http://dx.doi.org/10.1590/S0034-71672010000600009.

18. de Freitas DF, Oda JY. Avaliação dos fatores de risco relacionados às falhas durante a administração de medicamentos. Arq. Ciênc. Saúde Unipar. [Internet] 2008; 12(3) [acesso em 04 mai 2015]. Disponível: http://revistas.unipar.br/?journal=saude\&page=article\&op=view\&path[]=2540\&path[]=1983.

19. Manenti S, Chaves AB, Leopoldino RS, Padilha KG. Ocorrências adversas com medicação em unidade de terapia intensiva: análise da administração de soluções hidroeletrolíticas e antibióticos. Rev. esc. enferm. USP. [Internet] 1998; 32(4) [acesso em 04 mai 2015]. Disponível: http://dx.doi.org/10.1590/S0080-62341998000400010.

20. Silva AEBC, Reis AMM, Miasso AI, Santos JO, Cassiani SHB. Eventos adversos a medicamentos em um hospital sentinela do Estado de Goiás, Brasil. Rev. Latino-Am. Enfermagem. [Internet] 2011; 19(2) [acesso em 04 mai 2015 ]. Disponível: http://dx.doi.org/10.1590/S0104-11692011000200021.

21. de Souza LO, Carvalho APS, Chianca TCM, Freitas MEA, Ricaldon CAC. Classificação de erros de medicação ocorridos em um hospital privado de belo horizonte. Reme, Rev. Min. Enferm. [Internet] 2000; 4(1/2) [acesso em 04 mai 2015]. Disponível: http://www.reme.org.br/artigo/detalhes/822.

22. Teixeira TCA, Cassiani SHB. Análise de causa raiz: avaliação de erros de medicação em um hospital universitário. Rev. esc. enferm. USP. [Internet] 2010; 44(1) [acesso em 04 mai 2015]. Disponível:

http://dx.doi.org/10.1590/S0080-62342010000100020.

23. Sahay A, Hutchinson M, East L. Exploring the influence of workplace supports and relationships on safe medication practice: A pilot study of Australian graduate nurses. Nurse Educ Today. [Internet] 2015; 35(5) [acesso em 04 mai 2015]. Disponível: http://dx.doi.org/10.1016/j.nedt.2015.01.012.

24. Scott BM, Considine J, Botti M. Medication errors in ED: Do patient characteristics and the environment influence the nature and frequency of medication errors?. Australas Emerg Nurs J. [Internet] 2014; 17(4) [acesso em 04 mai 2015]. Disponível: http://dx.doi.org/10.1016/j.aenj.2014.07.004.

25. Kim KS, Kwon SH, Kim JA, Cho S. Nurses perceptions of medication errors and their contributing factors in South Korea. J. Nurs. Manag. [Internet] 2011; 19(3) [acesso em 04 mai 2015]. Disponível:

http://dx.doi.org/10.1111/j.1365-2834.2011.01249.x.

26. Desai RJ, Williams CE, Greene SB, Pierson S, Caprio AJ, Hansen RA. Exploratory evaluation of medication classes most commonly involved in nursing home errors. J. Am. Med. Dir. Assoc. [Internet] 2013; 14(6) [acesso em 04 mai 2015]. Disponível: http://dx.doi.org/10.1016/j.jamda.2012.11.006.

27. Hicks RW, Becker SC, Windle PE, Krenzischek DA. Medication Errors in the PACU. J. Perianesth. Nurs. [Internet] 2007; 22(6) [acesso em 04 mai 2015]. Disponível: http://dx.doi.org/10.1016/j.jopan.2007.08.002.

28. Husch M, Sullivan C, Rooney D, Barnard C, Fotis M, Clarke J, et al. Insights from the sharp end of intravenous medication errors: implications for infusion pump technology. Qual. Saf. Health Care. [Internet] 2005; 14(2) [acesso em 04 mai 2015]. Disponível: http://dx.doi.org/10.1136/qshc.2004.011957. 
29. Härkänen M, Turunen H, Saano S, Vehviläinen-Julkunen K. Detecting medication errors: Analysis based on a hospital's incident reports. Int. J. Nurs. Pract. [Internet] 2015; 21(2) [acesso em 04 mai 2015]. Disponível: http://dx.doi.org/10.1111/ijn.12227.

30. Joolaee S, Hajibabaee F, Peyrovi H, Haghani H, Bahrani N. The relationship between incidence and report of medication errors and working conditions. Int. Nurs. Rev. [Internet] 2011;58(1) [acesso em 04 mai 2015]. Disponível: http://dx.doi.org/10.1111/j.1466-7657.2010.00872.x.

31. Pazokian M, Tafreshi MZ, Rassouli M. Iranian nurses' perspectives on factors influencing medication errors. Int. Nurs. Rev. [Internet] 2014; 61(2) [acesso em 04 mai 2015]. Disponível: http://dx.doi.org/10.1111/inr.12086.

32. Fleming S, Brady AM, Malone AM. An evaluation of the drug calculation skills of registered nurses. Nurse. Educ. Pract. [Internet] 2014; 14(1) [acesso em 04 mai 2015]. Disponível: http://dx.doi.org/10.1016/j.nepr.2013.06.002.

33. Drach-Zahavy A, Pud D. Learning mechanisms to limit medication administration errors. J. Adv. Nurs. [Internet] 2010; 66(4) [acesso em 04 mai 2015]. Disponível: http://dx.doi.org/10.1111/j.1365-2648.2010.05294.x.

34. Choo J, Johnston L, Manias E. Nurses' medication administration practices at two Singaporean acute care hospitals. Nurs Health Sci. [Internet] 2013; 15(1) [acesso em 04 mai 2015]. Disponível: http://dx.doi.org/10.1111/j.1442-2018.2012.00706.x.

35. Armutlu M, Foley M, Surette J, Belzile E, McCusker J. Survey of nursing perceptions of medication administration practices, perceived sources of errors and reporting behaviours. Healthc Q. [Internet] 2008; 11(n.esp) [acesso em 04 mai 2015]. Disponível: http://dx.doi.org/10.12927/hcq.2008.19651.

36. Paranaguá TTB, Bezerra ALQ, dos Santos ALM, Silva AEBC. Prevalência e fatores associados aos incidentes relacionados à medicação em pacientes cirúrgicos. Rev. esc. enferm. USP. [Internet] 2014; 48(1) [acesso em 04 mai 2015]. Disponível: http://dx.doi.org/10.1590/S0080-623420140000100005.

37. da Silva LD, Camerini FG. Análise da administração de medicamentos intravenosos em hospital da rede sentinela. Texto Contexto Enferm. [Internet] 2012; 21(3) [acesso em 04 mai 2015]. Disponível:

http://dx.doi.org/10.1590/S0104-07072012000300019.

38. Aragão J. Introdução aos estudos quantitativos utilizados em pesquisas científicas. Rev. Práxis. [Internet] 2011; 3(6) [acesso em 24 mai 2015]. Disponível: http://web.unifoa.edu.br/praxis/ojs/index.php/praxis/article/view/35/28.

39. Silva ES, Pereira MGN, Munhoz CJMR. Os erros de medicação cometidos pelo profissional de enfermagem: como atuar nessa questão?. Rev. Int. Saúde. 2014; 6(6) 633-41.

40. Lopes LN, Garcia KP, Dias LG, Soares LR, Leite AM, da Silva JAC. Qualidade das prescrições médicas em um centro de saúde escola da Amazônia brasileira. Rev. Soc. Bras. Clín. Méd. [Internet] 2014; 12(2) [acesso em 24 mai 2015]. Disponível: http://files.bvs.br/upload/S/1679-1010/2014/v12n2/a4184.

41. Camerini FG, Colcher AP, Moraes DS, Souza DL, Vasconcelos JR, Neves RO. Fatores de risco para ocorrência de erro no preparo de medicamentos endovenosos: uma revisão integrativa. Cogitare Enferm. [Internet] 2014; 19(2) [acesso em 11 abr 2016]. Disponível: http://dx.doi.org/10.5380/ce.v19i2.37362.

42. Perehudoff K, Azermai M, Somers A, Stichele RV, Petrovic M. Medication discrepancies in older patients admitted to non-geriatric wards: An exploratory study. Eur. Geriatr. Med. [Internet] 2015; 6(1) [acesso em 04 mai 2015]. Disponível: http://dx.doi.org/10.1016/j.eurger.2014.10.005.

43. Gokhmana R, Seybert AL, Phrampusd P, Darbyf J, Kane-Gill SL. Medication errors during medical emergencies in a large, tertiary care, academic medical center. Resuscitation. [Internet] 2012; 83(4) [acesso em 04 mai 2015]. Disponível: http://dx.doi.org/10.1016/j.resuscitation.2011.10.001.

44. FitzHenry F, Peterson JF, Arrieta M, Waitman LR, Schildcrout JS, Miller RA. Medication Administration Discrepancies Persist Despite Electronic Ordering. J. Am. Med. Inform. Assoc. [Internet] 2007; 14(6) [acesso em 04 mai 2015]. Disponível: http://dx.doi.org/10.1197/jamia.M2359.

45. Young HM, Gray SL, McCormick WC, Sikma SK, Reinhard S, Trippett LJ, et al. Types, prevalence, and potential clinical significance of medication administration errors in assisted living. J Am Geriatr Soc. [Internet] 2008; 56(7) [acesso em 04 mai 2015]. Disponível: http://dx.doi.org/10.1111/j.1532-5415.2008.01754.x. 
46. Trbovich PL, Pinkney S, Cafazzo JA, Easty AC. The impact of traditional and smart pump infusion technology on nurse medication administration performance in a simulated inpatient unit. Qual. Saf. Health Care. [Internet] 2010; (19) [acesso em 04 mai 2015]. Disponível: http://dx.doi.org/10.1136/qshc.2009.032839.

47. Wang HF, Jin JF, Feng XQ, Huang X, Zhu LL, Zhao XY, et al. Quality improvements in decreasing medication administration errors made by nursing staff in an academic medical center hospital: a trend analysis during the journey to Joint Commission International accreditation and in the post-accreditation era. Ther. Clin. Risk Manag. [Internet] 2015; (11) [acesso em 04 mai 2015]. Disponível: http://dx.doi.org/10.2147/TCRM.S79238.

48. Berdot S, Sabatier B, Gillaizeau F, Caruba T, Prognon P, Durieux P. Evaluation of drug administration errors in a teaching hospital. BMC Health Serv. Res. [Internet] 2012; (12) [acesso em 04 mai 2015]. Disponível: http://dx.doi.org/10.1186/1472-6963-12-60.

49. van den Bemt PMLA, Idzinga JC, Robertz H, Kormelink DG, Pels N. Medication administration errors in nursing homes using an automated medication dispensing system. J. Am. Med. Inform. Assoc. [Internet] 2009;16(4) [acesso em 04 mai 2015]. Disponível: http://dx.doi.org/10.1197/jamia.M2959.

50. Lisboa CD, da Silva LD, de Matos GC. Investigação da técnica de preparo de medicamentos para administração por cateteres pela enfermagem na terapia intensiva. Rev. esc. enferm. USP. [Internet] 2013; 47(1) [acesso em 21 jul 2015]. Disponível: http://dx.doi.org/10.1590/S0080-62342013000100007.

51. Ferreira $M M M$, Jacobina FMB, Alves FS. O profissional de enfermagem e a administração segura de medicamentos. Revista Enfermagem Contemporânea. [Internet] 2014; 3(1) [acesso em 21 jul 2015]. Disponível: http://dx.doi.org/10.17267/2317-3378rec.v3i1.208.

52. Conselho Regional de Enfermagem do Estado de São Paulo (COREN-SP). Rede Brasileira de Enfermagem e Segurança do Paciente (REBRAENSP). Erros de medicação definições e estratégias de prevenção. [Internet] São Paulo: COREN-SP/ REBRAENSP; 2011 [acesso em 24 mai 2015]. Disponível: http://www.rebraensp.com.br/ phocadownload/publicacoes/erros_de_medicacao-definicoes_e_estrategias_de_prevencao.pdf.

53. Westbrook JI, Rob MI, Woods A, Parry D. Errors in the administration of intravenous medications in hospital and the role of correct procedures and nurse experience. BMJ Qual. Saf. [Internet] 2011; 20(12) [acesso em 04 mai 2015]. Disponível: http://dx.doi.org/10.1136/bmjqs-2011-000089.

54. Elliott M, LiuY. The nine rights of medication administration: an overview. Br. J. Nurs. [Internet] 2010; 19(5) [acesso em 21 jul 2015]. Disponível: http://dx.doi.org/10.12968/bjon.2010.19.5.47064.

55. Rossetti AC, Gaidzinski RR. Estimativa do quadro de pessoal de enfermagem em um novo hospital. Rev. Latino-Am. Enfermagem. [Internet] 2011; 19(4) [acesso em 24 mai 2015]. Disponível:

http://dx.doi.org/10.1590/S0104-11692011000400021.

56. Keers RN, Williams SD, Cooke J, Ashcroft DM. Causes of medication administration errors in hospitals: a systematic review of quantitative and qualitative evidence. Drug Saf. [Internet] 2013; 36(11) [acesso em 04 mai 2015]. Disponível: http://dx.doi.org/10.1007/s40264-013-0090-2.

56. Arboit EL, da Silva LAA. Eventos adversos relacionados à terapia medicamentosa na enfermagem. Revista de Enfermagem. [Internet] 2012; 8(8) [acesso em 24 mai 2015]. Disponível: http://revistas.fw.uri.br/index.php/ revistadeenfermagem/article/view/482/878.

57. Conselho Federal de Enfermagem. Resolução n. 311, de 08 de fevereiro de 2007. Aprova a Reformulação do Código de Ética dos Profissionais de Enfermagem. Rio de Janeiro: COFEN; 2007. 